\title{
High Performance Transistors in Low Mobility Organic Semiconductors for Analog and High-Frequency Applications
}

\author{
Xiaojun Guo*, Frantisek Balon, Ross A. Hatton, and J. M. Shannon \\ Advanced Technology Institute, University of Surrey, \\ Guildford, GU2 7XH, United Kingdom \\ *Email: x.guo@surrey.ac.uk Tel: +44 (0)1483 686083 Fax: +44 (0) 1483689404
}

\begin{abstract}
In conventional organic field-effect transistors (OFETs), the low mobility of carriers in the organic semiconductor layers leads to low device speed, which seriously limits the applicability of the devices for analog and high frequency circuits in high performance applications. In this paper, the sourcegated transistor (SGT) concept is introduced for high performance transistors in low mobility organic semiconductors, in which the source comprises a potential barrier to current flow and a gate is used to modulate the electric field at the reverse-biased source barrier and change the current. Compared to the OFET, the OSGT has a much smaller susceptibility to short-channel effects, and can be operated with much higher internal electrical fields giving high drive current and high speed. The numerical simulation results show that the OSGT can be an excellent candidate for designing high performance transistors in low-mobility organic materials for analog and high frequency applications.
\end{abstract}

\section{Keywords}

Organic semiconductor, thin-film transistor (TFT), source-gated transistor, short-channel effects, fielddependent mobility, frequency response

\section{Introduction}

There has been rapid acceleration recently in the research and development of organic field-effect transistors (OFETs) as key components for displays, radio frequency identification tags, and some other small-scale integrated circuits owing to their low cost and flexible properties $[1,2]$. However, the main limitation to extensive exploitation of OFETs comes from their low mobility which is, at best, two or three orders of magnitude lower than that of standard semiconductors. Carrier transport in organic semiconductors depends on hopping or tunnelling between localized states [3]. Since carriers spend a significant amount of time trapped in states, the effective mobility is low and as a result the transistor speed is reduced, leading to a severe limitation of analog and high frequency performance. A considerable amount of basic research in the past is aimed at improving the quality of organic material, particularly by reducing the amount of disorder to reduce trap depths and improve mobility [4]. An alternative approach to improve transistor speed is to design structures with high internal electrical fields, because firstly, for a given mobility, carrier velocity is proportional to electric field and secondly in many organic semiconductors the mobility itself is field dependent $[3,5]$. In material systems with disorder, the electric field required to produce significant mobility changes is high, being $10^{5} \mathrm{~V} / \mathrm{cm}$ at room temperature [3]. This requires the design of short channel OFETs. However, in this case, achieving thin gate insulator layers of high quality to suppress the short-channel effects will be difficult. Here, the proposed source-gated transistor (SGT) can be designed to operate with much higher internal electrical fields than the conventional FET, and brings the possibility of designing high performance circuits in low-mobility organic material for analog and high frequency applications.

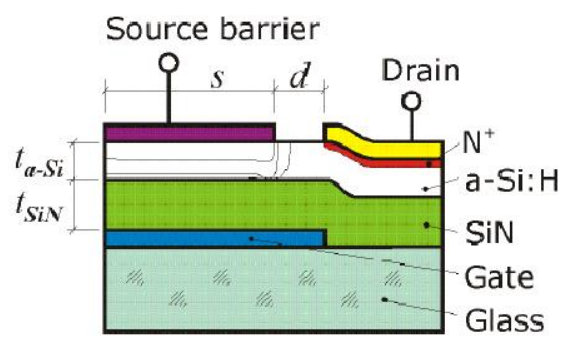

Fig. 1 Schematic drawing of a SGT structure in amorphous silicon (a-Si:H) [6].

\section{Operation of Source-Gated Transistor}

The SGT concept was first introduced in [6] for amorphous silicon transistors. As shown in Fig. 1, in the SGT, the source is a potential barrier to current transport and the drain is assumed to be ohmic. The gate lies under the source barrier and extends under the source-drain separation $d$ to the drain contact. Although a doped layer is used to make a drain ohmic contact, it is not essential. When the transistor is switched on, the current in the SGT is determined by the carrier emission over the source-barrier 
contact [7], and the channel forms a parasitic resistance. Most of the SGT current flows at the periphery of the source barrier opposite to the drain [8]. Details of the operation of the SGT have been described previously [6-9].

A major difference between the SGT and a FET is that a reverse-biased source barrier controls the current in the ON-state and current saturation is determined by the electrostatics at the source barrier rather than pinch-off at the drain end of a channel. In addition, the geometry of the SGT leads to a much smaller susceptibility to short-channel effects and a higher output impedance because the source barrier is screened from the drain field by the gate [9]. What is interesting in the context of our requirements for high-performance transistors in low mobility organic semiconductors is that there are high fields sweeping the carriers away toward the drain. Furthermore, these transistors operate very well with small dimensions.

\section{Simulation Method}

To compare the performance of SGT and FET in organic semiconductors, the OSGT and OFET structures, as shown in Fig. 2, were simulated using the two-dimensional Atlas device simulator vended by Silvaco [10]. The usual values of pentacene (energy gap of $2.5 \mathrm{eV}$, ionization potential of $5 \mathrm{eV}$ and dielectric constant of 4.0 ) have been used for the organic semiconductors in both structures [11]. The field-dependent mobility of holes is described by the Poole-Frenkel model $[3,5]$, as:

$$
\mu=\mu_{0} e^{\sqrt{E / E_{0}}}
$$

where $\mu_{0}$ is the low field mobility, and $E_{0}$ is a characteristics parameter equal to $3 \times 10^{5} \mathrm{~V} / \mathrm{cm}$. The effective density of the states $\left(N_{\mathrm{V}}\right)$ is set to be $10^{19}$ $\mathrm{cm}^{-3}$. Simulations based on these models and parameters have been shown to give good fits to the experimental data for OFETs [12].

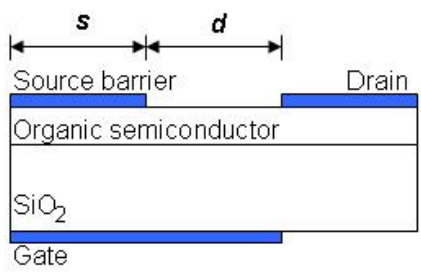

(a)

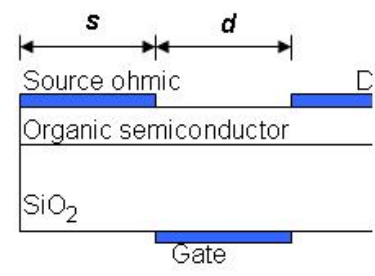

(b)
Fig. 2 The device structures used for the simulations: (a) SGT; (b) FET.

In the OSGT, a work function of $4.55 \mathrm{eV}$ has been taken for the source contact to produce a barrier of $0.45 \mathrm{eV}$ height for hole transport. The drain is assumed to be ohmic contact. And in the OFET, both contacts are ohmic. A $15 \mathrm{~nm}$ thick channel, and an 80 $\mathrm{nm}$ thick gate insulator $\left(\mathrm{SiO}_{2}\right)$ are used in both structures. The channel width is $1 \mu \mathrm{m}$. The source length is fixed as $0.5 \mu \mathrm{m}$. For the OSGT, the change of effective source barrier height was assumed to be proportional to the electric field [13].

\section{Results and Discussion}

\subsection{Susceptibility to short-channel effects}

High output impedance, high drive current and high speed are important for transistors in analog and high-frequency applications. For OFETs, a shortchannel for high internal field is preferred for achieving high drive current and high speed, but poses difficulties in suppressing short-channel effects that degrade the output impedance. In the SGT, since the saturation current is determined by the electrostatics at the source barrier, and the source barrier is screened from the drain field by the gate, the susceptibility to short-channel effects is much weaker. This feature of the SGT enables it to operate with very short source-drain separations even at a thick gate insulator lay er.

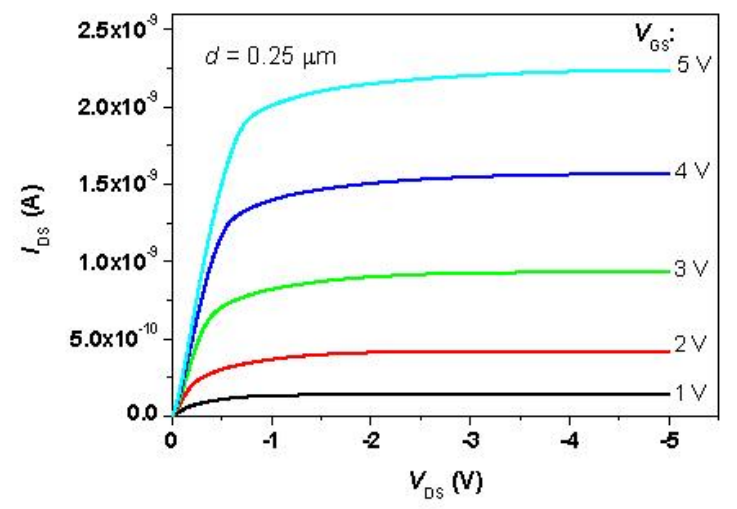

(a) 


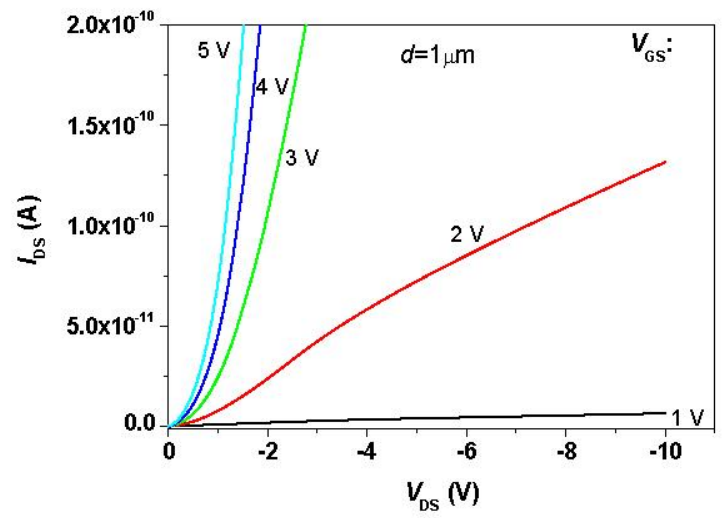

(b)

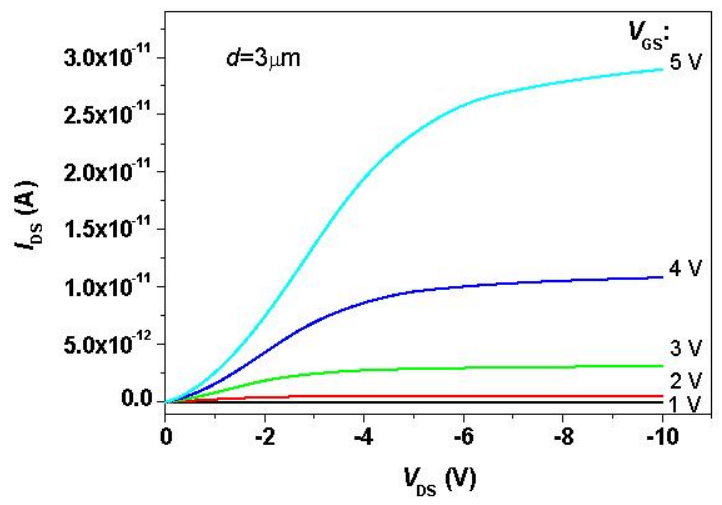

(c)

Fig. 3 The simulated output characteristics $\left(I_{\mathrm{DS}}-V_{\mathrm{DS}}\right)$ for (a) an OSGT with $d=0.25 \mu \mathrm{m}$, (b) the OFET with $d=1.0 \mu \mathrm{m}$, and (c) the OFET with $d=3.0 \mu \mathrm{m}$. $\mu_{0}=0.001 \mathrm{~cm}^{2} / \mathrm{V} \cdot \mathrm{s}$.

As seen in Fig. 3, with a $15 \mathrm{~nm}$ thick channel and a $80 \mathrm{~nm}$ thick $\mathrm{SiO}_{2}$ insulator layer, the OSGT can worked at a source-drain separation (d) of as small as $0.25 \mu \mathrm{m}$ with extremely good output characteristics, while the channel length of a FET must be extended to $3 \mu \mathrm{m}$ to get saturated output characteristics, which agrees with the experimental results in [3]. This indicates a major advantage of SGT for high-speed organic electronics, since in terms of fabrication processes, it is still a big hurdle to produce the ultrathin, high quality gate insulator layer needed for short-channel FETs, while short-channel SGTs can work well with a thicker insulator layer.

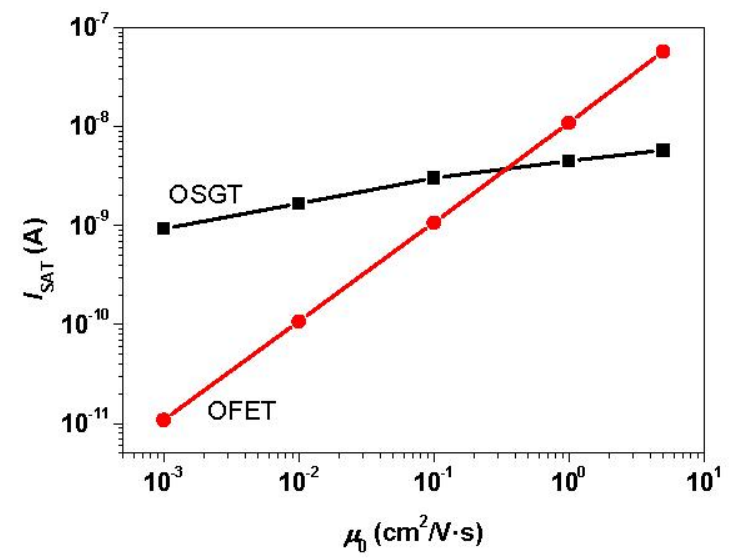

Fig. 4 The simulated drain saturation current $\left(I_{\mathrm{SAT}}\right)$ in an OSGT and an OFET as a function of the low field mobility $\mu_{0}$, showing a much reduced dependence of drive current in an OSGT compared with an OFET. The gate bias for both structures is $4 \mathrm{~V}$.

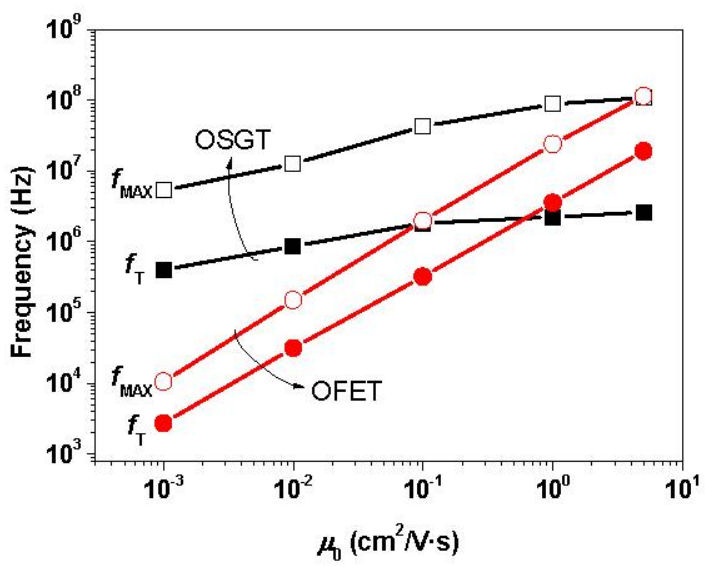

Fig. 5 The simulated cut-off frequency $\left(f_{\mathrm{T}}\right)$ and maximum oscillation frequency $\left(f_{\mathrm{MAX}}\right)$ in an OSGT and an OFET, showing the much reduced dependence on $\mu_{0}$ of the OSGT. The drain bias is $4 \mathrm{~V}$ for the OSGT, and $8 \mathrm{~V}$ for the OFET, with a gate bias of $4 \mathrm{~V}$ for both structures.

\subsection{Drive current and frequency response}

As discussed in [14], the way to obtain high drive current and a good frequency response for a given geometry transistor despite having a low carrier mobility is to operate with high internal fields over short distances to reduce carrier transit times combined with low carrier concentrations for low capacitance. The simulations show that the electric field along the interface for the SGT at the edge of 
the source, where most of the current flows [8], is as much as six times greater than in the corresponding FET [14]. Because both the carrier velocity and charge mobility are field-dependent in the organic material, much higher internal electric field in OSGT brings higher drive current than that in the OFET when the low field mobility is small, as shown in Fig. 4. With a $\mu_{0}$ of $0.001 \mathrm{~cm}^{2} / \mathrm{V} \cdot \mathrm{s}$, the OSGT can achieve a drive current 100 times that of an OFET at a gate bias of $4 \mathrm{~V}$. When $\mu_{0}$ is large, the saturation current in the OSGT is limited by hole emission over the source barrier, and thus becomes lower than an equivalent $\mathrm{OFET}$.

The frequency response of OSGT and OFET were also simulated using the Atlas device simulator. Similarly, much higher cut-off frequency $\left(f_{\mathrm{T}}\right)$ and maximum oscillation frequency $\left(f_{\text {MAX }}\right)$ are achieved by the SGT structure in low $\mu_{0}$ material. With a $\mu_{0}$ of $0.001 \mathrm{~cm}^{2} / \mathrm{V} \cdot \mathrm{s}$, the OSGT has a $f_{\mathrm{T}}$ about $0.4 \mathrm{MHz}$, which is more than 100 times of the value for the OFET. To obtain a higher $f_{\mathrm{T}}$, we can operate the OSGT with a higher gate bias, and can also reduce the barrier height at the source contact. We found, reducing the OSGT source barrier height by $65 \mathrm{meV}$, for example, would increase $f_{\mathrm{T}}$ by approximately an order of magnitude. Thus, the OSGT may enable transistors in very low mobility organics for high frequency applications. Although with higher $\mu_{0}$, the FET can achieve a higher $f_{\mathrm{T}}$ due to higher drive current, the $f_{\mathrm{MAX}}$ of the OFET is still lower than that of the OSGT, which is because the OSGT has much better output saturation characteristics.

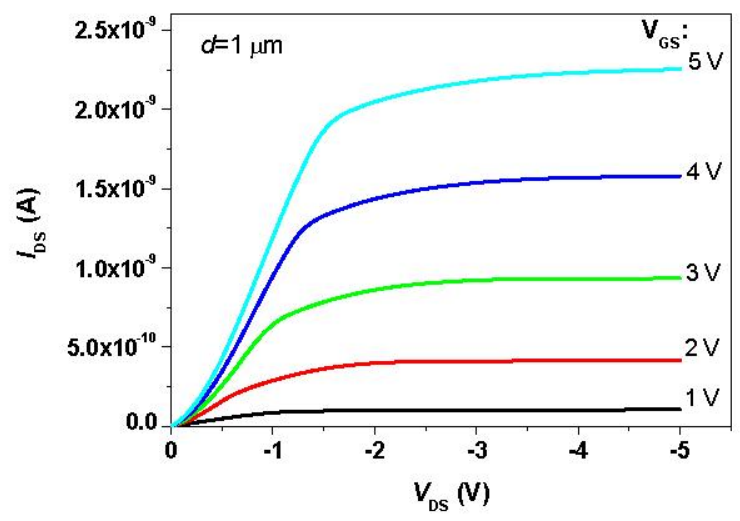

(a)

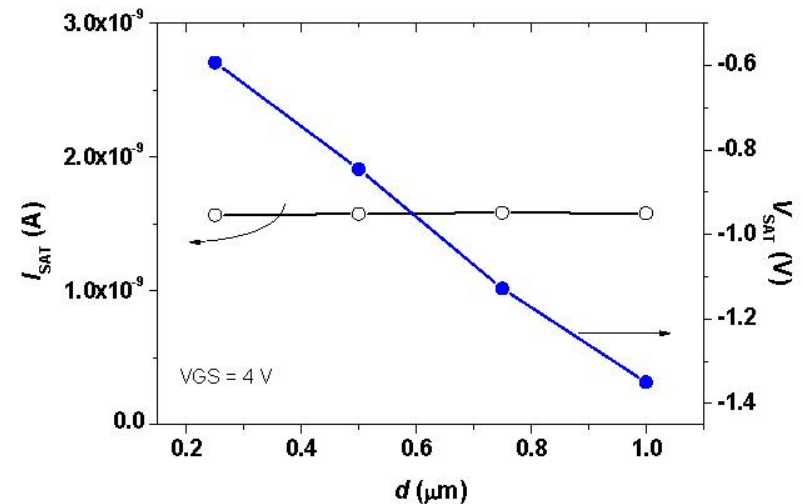

(b)

Fig. 6 (a) The output characteristics $\left(I_{\mathrm{DS}}-V_{\mathrm{DS}}\right)$ of OSGT with $d=1 \mu \mathrm{m}$, and $\mu_{0}=0.001 \mathrm{~cm}^{2} / \mathrm{V} \cdot \mathrm{s} ;$; (b) $I_{\mathrm{SAT}}$ and the drain saturation voltage $\left(V_{\mathrm{SAT}}\right)$ as a function of $d$ with $\mu_{0}=0.001 \mathrm{~cm}^{2} / \mathrm{V} \cdot \mathrm{s}$.

The results in Fig. 4 and Fig. 5 show the drive current and speed of OSGT are much less sensitive to the low-field the mobility of the channel material than that of the OFET, thus making the SGT a more suitable candidate for achieving high performance transistors in low mobility organic semiconductors.

\subsection{Source-drain separation on OSGT performance}

High-speed performance of the OSGT needs small source-drain separation. Through simulating OSGTs with different values of $d$, we found that the drive current is insensitive to the source-drain separation (Fig. 6). As shown in Fig. 6 (a), for an OSGT with $d=1 \mu \mathrm{m}$, the current levels in the saturation regime at different $V_{\mathrm{GS}}$ are quite close to that of an OSGT with $d=0.25 \mu \mathrm{m}$ shown in Fig. 3 (a). As mentioned above, this is because, in the SGT, the saturation current is determined by the carrier emission over the source-barrier contact, while the barrier is mainly controlled along the vertical direction. However, as $d$ increases, the drain voltage that is required to deplete the channel is increased, so the absolute value of saturation voltage $\left(V_{\mathrm{SAT}}\right)$ increases approximately linearly with $d$ as shown in Fig. 6(b). The results are important because if the current is insensitive to source-drain separation, the technology used does not need to provide precisely defined gaps between the source and drain regions. For example, fabrication of submicron source-drain separations can be achieved using self-aligned spacer technology, since the separations are similar to standard layer thicknesses. And the exact control of these thicknesses is not necessary for making OSGTs 
with good current uniformity. Similarly, an imprecise dewetting technology [15] is also much better suited to an OSGT than an OFET.

\section{Conclusions}

Compared to a FET, the ON-state current in the SGT is controlled by source barrier and current saturation is determined by the electrostatics at the source barrier rather than pinch-off at the drain end of a channel. This feature enables SGTs to operate with very short source-drain separations even with a thick gate insulator layer, thus providing a big advantage in terms of the fabrication process. The SGT structure can also be operated with much higher internal electric fields to increase the carrier velocity in organic materials for high drive current and frequency response that can be orders of magnitude higher than an OFET. It is shown that the current through an OSGT is insensitive to source-drain separation thus enabling current uniformity to be obtained with simple and imprecise technology. It is concluded that, the SGT is an excellent candidate for building high performance organic electronics for analog and high frequency applications.

\section{Acknowledgments}

The authors would like to thank EPSRC, UK for funding this project. Dr Ross A. Hatton is a Royal Academy of Engineering/EPSRC Research Fellow.

\section{References}

1. S. R. Forrest, "The path to ubiquitous and lowcost organic electronic appliances on plastic," Nature, Vol. 428, No. 6986, pp. 911-918, Apr. 2004.

2. B. Crone, et al, "Large-scale complementary integrated circuits based on organic transistors", Nature. Vol. 403, No. 6769 , pp. 521-523, Feb. 2000.

3. L. Wang, D. Fine, D. Basu, A. Dodabalapur, " Electric-field-dependent charge transport in organic thin-film transistors", J. Appl. Phys., Vol. 101, pp. 054515-1, 2007.

4. C. D. Dimitrakopoulos, D. J. Mascaro, "Organic thin- film transistors: review of recent advances", IBM J. Res. \& Dev., Vol. 45, No. 1, pp. 11-27, 2001.

5. G. Horowitz, "Organic Field-Effect Transistors", Advanced Materials, Vol. 10, No.5, pp. 365-377, 1998.

6. J. M. Shannon and E. G. Gerstner, "Source-gated thin-film transistors," IEEE Electron Device Lett., Vol. 24, No. 6, pp. 405-407, June 2003.

7. F. Balon, J. M. Shannon, "Analysis of Schottky barrier source-gated transistors in a-Si:H", Solid State Electron., Vol. 50, No. 3, pp. 378-383, Mar. 2006.

8. — "Modeling of source-gated transistors in amorphous silicon", J. Electrochem. Soc., Vol. 152, No. 8, pp. G674-G677, 2005.

9. J. M. Shannon, E. G. Gerstner, "Source-gated transistors in hydrogenated amorphous silicon", Solid State Electron., vol. 48, No. 7, pp. 11551161, Jul. 2004.

10. ATLAS User's Manual: Silvaco International, Inc., 2005.

11. T. Li, J.W. Balk, P. P. Ruden, I. H. Campbell, and D. L. Smith, "Channel formation in organic field-effect transistors," J. Appl. Phys., Vol. 91, pp. 4312-4318, Apr. 2002.

12. A. Bolognesi, et al, "Effects of grain boundaries, field dependent mobility, and interface trap states on the electrical characteristics of Pentacene TFT", IEEE Trans. Elec. Dev., Vol. 51 , No. 12, pp. 1997-2003, Dec. 2004.

13. A. K. Mahapatro, and S. Ghosh, "Schottky energy barrier and charge injection in metal/copper-phthalocyanine /metal structures", Appl. Phys. Lett., Vol. 80, pp. 4840-4842, June, 2002.

14. J. M. Shannon and F. Balon, "High-performance thin-film transistors in disordered and poorquality semiconductors", IEEE Trans. Elec. Dev., Vol. 54 , No. 2, pp. 354-358, Feb. 2007. 15. J.Z. Wang, Z.H. Zheng, H.W. Li, W.T.S. Huck, and H. Sirringshaus, "Polymer field effect transistors fabricated by dewetting", Synthetic Metals, Vol. 146., pp. 287-290, 2004. 